\title{
Producing Jatropha oil-based polyol via epoxidation and ring opening
}

\begin{abstract}
A low viscosity polyol has been functionalized from crude Jatropha oil via epoxidation and subsequent ring-opening. Starting with the crude Jatropha oil, the double bonds are functionalized by introducing epoxy groups and ring-opened to produce hydroxyl groups. The experiment employs more concentrated $50 \%$ hydrogen peroxide and effectively produce solvent-free epodixidized Jatropha oil within shorter reaction time of $5 \mathrm{~h}$ with maximum oxirane oxygen content of $4.30 \%$ and viscosity of $0.57-0.60$ Pa.s. The epoxidized Jatropha oil is then transform into Jatropha-based polyol with hydroxyl number of 171-179 mg $\mathrm{KOH} / \mathrm{g}$, low viscosity of $0.92-0.98 \mathrm{~Pa}$.s. and functionality of 5.1-5.3. The epoxidation and ring-opening process are monitored by viscometer and FTIR. The produced polyol permit more time for molding and additives addition during polyurethane due to its low viscosity.
\end{abstract}

Keyword: Epoxidation; Hydroxylation; Renewable polyol 